\title{
Characteristics of Patients with SARS-CoV-2 Positive Cerebrospinal Fluid: A Systematic Review
}

\author{
Maryam A Salman (D) \\ Saad I Mallah (D) \\ Wasay Khalid (iD ${ }^{\prime}$ \\ Laura Ryan Moran (1D) \\ Yousef Al Abousedu (iD ${ }^{3}$ \\ Ghufran A Jassim (D) ${ }^{4}$ \\ 'School of Medicine, Royal College of \\ Surgeons in Ireland, Busaiteen, Kingdom \\ of Bahrain; ${ }^{2}$ School of Medicine, Royal \\ College of Surgeons in Ireland, Dublin, \\ Ireland; ${ }^{3}$ Department of Neurosurgery, \\ Ibn Sina Hospital, Kuwait City, Kuwait; \\ ${ }^{4}$ Department of Family Medicine, Royal \\ College of Surgeons in Ireland, Busaiteen, \\ Kingdom of Bahrain
}

Correspondence: Ghufran A Jassim Department of Family Medicine, Royal College of Surgeons in Ireland, Busaiteen, Kingdom of Bahrain

Tel +97316660I 25

Email gjassim@rcsi-mub.com
Background: The coronavirus disease 2019 (COVID-19) has been shown to affect several systems, notably the respiratory system. However, there has been considerable evidence implicating the nervous system in COVID-19 infection. This study aims to investigate the clinical characteristics of patients whose cerebrospinal fluid (CSF) tested positive for SARS-CoV-2.

Methods: A comprehensive search of PubMed, EMBASE, Scopus, WHO Coronavirus database, bioRxiv, medRxiv, and Web of Science databases was carried out in August 2020. Original studies involving patients who tested positive for SARS-COV-2 in their CSF were included. Key search terms encompassed all variations of "COVID-19" AND "Cerebrospinal Fluid".

Results: A total of 525 studies were identified. Fifty-six full-text articles were assessed, of which 14 were included. In total, 14 patients tested positive for SARS-CoV-2 in their CSF. $21.4 \%$ (3/14) of patients had negative nasopharyngeal (NP) swabs despite a positive CSF sample. About $14.2 \%$ (2/14) of patients who initially had positive NP swabs developed neurological deterioration after a supposed recovery as indicated by their negative NP swabs, but their CSF still tested positive for SARS-CoV-2. Common symptoms were headache $(42.8 \% ; 6 / 14)$, fever $(35.6 \% ; 5 / 14)$, vomiting $(28.6 \% ; 4 / 14)$, cough $(28.6 ; 4 / 14)$, visual disturbances $(28.6 \% ; 4 / 14)$, diarrhea $(21.4 \% ; 3 / 14)$, and seizures $(21.4 \% ; 3 / 14)$. Four patients $(28.6 \%)$ were admitted to ICU, one $(7.14 \%)$ was admitted to a rehabilitation facility, and two (14.3\%) died.

Conclusion: Physicians should be familiar with the presenting neurological features of COVID-19, and be aware that they can occur despite a negative NP swab. The results of this study are intended to aid in the development of informed guidelines to diagnose and treat COVID-19 patients with neurological manifestations.

Keywords: COVID-19, SARS-CoV-2, CNS, CSF, central nervous system, cerebrospinal fluid

\section{Background}

Coronavirus Disease 2019 (COVID-19) is a novel infectious disease capable of causing mild to severe illness, typically respiratory, in both humans and animals. The virus responsible for COVID-19, referred to as Severe Acute Respiratory Syndrome Coronavirus 2 (SARS-CoV-2), resides primarily in the respiratory tract and causes symptoms ranging from mild cough, sore throat, and nasal congestion to more severe respiratory distress. Recently, it has been shown to have additional neuro-invasive potential. ${ }^{1}$ Infected patients globally have been reported to have headaches, paraesthesia, anosmia, ageusia, neuralgia, and dizziness. ${ }^{2}$

Additionally, several case reports and cohort studies have reported rare cases of meningoencephalitis, seizures, and immune-mediated neurological diseases. ${ }^{3}$ 
SARS-CoV-2 can either infiltrate the peripheral nervous system (PNS) and migrate to the CNS or directly infect the CNS. ${ }^{4}$ There are three postulated mechanisms of transmission of the SARS-CoV-2 virus via the PNS: the transcribial route, axonal transport, and trans-synaptic transfer, and hematogenous and/or lymphatic route. ${ }^{5}$ The transcribial route involves a primary olfactory infection followed by infiltration into the subarachnoid space via the cribriform plates. ${ }^{4,6}$ The axonal transport and transsynaptic transfer hypothesis suggests that an initial infection of peripheral nerve terminals results in a migration of the virus, up the nervous system, to the trigeminal, olfactory, and/or vagus nerve. ${ }^{4,6}$ It is important to note that both the gastrointestinal and the respiratory branches of the vagus nerve are susceptible to the infection..$^{7-10}$ An infection of the CNS may occur via direct contact of the SARSCoV-2 virus with the brain microvascular endothelial cells. This in turn leads to extracellular virus release into the CNS parenchyma. Lastly, compromised tight junctions at the blood brain barrier or virally infected leukocytes may provide viral access to the CNS via endocytosis. ${ }^{9,10}$ More research is needed to accurately map the neurologic pathogenesis of SARS-CoV-2, and how this may translate to clinical diagnosis, prognosis, and patient care.

Dealing with a pandemic of this magnitude requires rapid and effective diagnostic tools to help combat the disease as early as possible. The diagnostic tool most widely accepted is the reverse transcriptase polymerase chain reaction (RT-PCR), used on a nasopharyngeal (NP) sample. ${ }^{11}$ Although SARS-CoV-2 RT-PCR is typically conducted on an NP swab, it can also be conducted on a cerebrospinal fluid (CSF) sample obtained from a lumbar puncture (LP). ${ }^{12-14}$

In order to best understand the pathophysiology of SARS-CoV-2 as it relates to neuropsychiatric manifestations, it is important to explore viral presence in the nervous system, and how this may correlate - if at allwith clinical presentation and outcomes. Thus, this systematic review aims to compile and synthesize primary studies that report on patients who tested positive for SARS-CoV-2 via their CSF sample. Our study investigates the unique clinical manifestations and characteristics of this patient cohort, along with relevant outcomes, disease progression and management. Furthermore, we hope our findings will help identify when to consider PCR CSF tests despite a negative NP swab test. ${ }^{15}$ By exploring the CNS involvement in SARS-CoV-2, this can aid in the development of new guidelines to diagnose and treat COVID-19 patients with neurological involvement.

\section{Methods}

\section{Eligibility}

We included primary research papers (case reports, case studies, cohort studies, cross-sectional studies, randomised control trials, letters to the editor reporting primary findings) that investigated the clinical course, outcomes, prognosis, management, and characteristics of patients who tested positive for SARS-COV-2 in their CSF using RTPCR test. Exclusion criteria included non-English articles, animal studies, and non-original articles (eg, editorials that did not contain original data).

\section{Search Strategy}

We conducted our search in PubMed NCBI, Excerpta Medica dataBASE, Scopus, WHO COVID-19 Global literature on coronavirus disease database, Biorxiv and Medrxiv, and Web of Science on August 24th, 2020 using the following search terms: (("Cerebrospinal fluid" [Mesh]) OR ("CSF" OR "Cerebrospinal fluid" OR "Cerebral spinal fluid" OR "Cerebro-spinal fluid" OR "Lumbar puncture" OR "Spinal tap")) AND ("coronavirus" [MeSH] OR "coronavirus infections" [MeSH Terms] OR "coronavirus" [All Fields] OR “covid 2019" [All Fields] OR "SARS2" [All Fields] OR "SARS-CoV-2" [All Fields] OR "SARS-CoV -19" [All Fields] OR "severe acute respiratory syndrome coronavirus 2" [supplementary concept] OR "coronavirus infection" [All Fields] OR "severe acute respiratory pneumonia outbreak" [All Fields] OR "novel cov" [All Fields] OR “2019ncov” [All Fields] OR "sars cov2" [All Fields] OR "cov22" [All Fields] OR "ncov" [All Fields] OR "covid-19" [All Fields] OR "covid19" [All Fields] OR "coronaviridae" [All Fields] OR "corona virus" [All Fields]). The selection criteria were limited to papers published from December 2019 until August 2020 and papers written in English.

\section{Study Selection}

After deduplication of the titles, two reviewers independently screened all the titles and abstracts of the papers according to the predefined inclusion and exclusion criteria. Next, full texts of potentially eligible studies were retrieved and reviewed independently by two authors. A third author resolved any disagreement. Reviews that included patients who tested positive for SARS-CoV-2 in 
their CSF were cross checked to identify any studies that matched our eligibility criteria.

\section{Data Extraction and Interpretation}

Data was extracted via a dual approach by two independent reviewers and inserted into a standardized review sheet. Data collected includes study characteristics (study title, authors, date of publication, publication type, study site, number of subjects), population characteristics, clinical findings, radiological findings, management, and final outcome. A third author resolved any disagreement.

\section{Risk of Bias in Individual Studies}

Two authors assessed the quality of the selected articles utilizing the National Institutes of Health (NIH) quality assessment tool for observational cohort and crosssectional studies. Quality assessment of case reports was carried out using Joanna Briggs Institute (JBI) critical appraisal checklist for case reports.

\section{Meta-Analysis}

A meta-analysis was not performed due to the preliminary nature of the study. Our aim was to review the literature in a scoping manner, and systemically gather and report the relevant data in the literature. In addition, due to the qualitative, heterogenic narrative nature of the outcomes, and the limited number of case reports (and absence of clinical studies), a meta-analysis would not be appropriate.

\section{Results}

\section{Study Selection}

An initial search of seven databases yielded a total of 525 publications. Fifty-six full-text articles were included and assessed for eligibility post abstract screening for relevance and deduplication, of which 14 were qualitatively analysed. After the application of the inclusion/exclusion criteria, they were narrowed down to 14. A Preferred Reporting Items for Systematic Reviews and Meta-Analyses (PRISMA) flow diagram explaining the steps of identification, screening, inclusion, and exclusion is presented in Figure 1.

\section{Study and Patients Characteristics}

Of the 14 articles included in this study, nine were case reports, ${ }^{10,16-23}$ three were retrospective studies, ${ }^{24-26}$ one was a letter to the editor reporting original data of a patient, ${ }^{27}$ and one follow-up letter to the editor of the same latter patient. ${ }^{28}$ All were published in 2020. The studies were conducted worldwide, including France, USA, Spain, Brazil, Japan, Turkey, Sweden, UAE, France, Germany, and Iran (Table 1). From the 14 eligible studies identified, the total sample size was 733 . Out of these, only 14 patients tested positive for SARS-CoV-2 in their CSF samples. As the scope of this review is to investigate only patients who tested positive for SARS-CoV-2 in their CSF according to the eligibility criteria, we only described these 14 patients. The mean age of the patients was $40(\mathrm{SD} \pm 15.7)$ and the median was 47.5 , with $50 \%$ of them being females. Comorbidities were present in $40 \%$ (4/10) of the patients, and were mainly hypertension (2/ $4),{ }^{16,22}$ ischemic heart disease (1/4), ${ }^{22}$ diabetes (1/4), ${ }^{28}$ metastatic colorectal cancer (1/4), ${ }^{22}$ migraines (1/4), ${ }^{16}$ and one patient had prior pancreatic-kidney transplant surgery $(1 / 4){ }^{23}$

\section{Clinical Course and Diagnosis}

In $21.4 \%$ (3/14) of cases, nasopharyngeal (NP) swabs initially tested negative despite a positive CSF sample. ${ }^{10,17,18} 14.2 \%$ $(2 / 14)$ of positive cases as per NP swab tested negative after supposed recovery but progressed to neurological deterioration and positive CSF tests. ${ }^{20,22} 10 / 14$ patients had both positive nasopharyngeal sample and CSF sample ${ }^{16,19-25,28}$ (in two of these cases CSF was not tested initially, but was found to be positive at post-mortem), however samples were not always positive on the first test; $3 / 14$ cases demonstrated a positive nasopharyngeal test but an initially negative CSF test. $^{20,22,28}$ Table 2 summarises the clinical and diagnostic findings.

\section{Symptoms}

Most commonly reported symptoms included: Headache $(6 / 14),{ }^{10,16,17,19,21,28}$ fever $(5 / 14),{ }^{17,20,21,23,28}$ vomiting (4/14), ${ }^{16,21}$ cough (4/14), ${ }^{10,19,21,23}$ visual disturbances (4/ $14),{ }^{16,19,23,28}$ diarrhoea $(3 / 14),{ }^{18,21,23}$ and seizure $(3 / 14)^{17,23,28}$ (Table 2). In two of the studies, the patients' COVID status was identified as severe ${ }^{25,26}$ and in one of these cases the patient was noted to be suffering from acute respiratory distress syndrome. ${ }^{25}$ Neurological symptoms were cited as the reason CSF test was carried out in $6 / 14$ of the studies. ${ }^{16-18,20,22,28}$

\section{Lab Findings}

Studies of the positive patients' CSF samples (Table 3) revealed leukocytosis in $2 / 14$ patients, ${ }^{17,22}$ elevated CSF protein (hyperproteinorrachia) in 3/14, ${ }^{22,23,28}$ hypoglycorrhachia in $1 / 14,{ }^{22}$ and an elevated red blood cells (RBCs) in $1 / 14$ samples. $^{28}$ D-dimers were elevated in $3 / 14$ blood samples. ${ }^{16,21,22}$ 


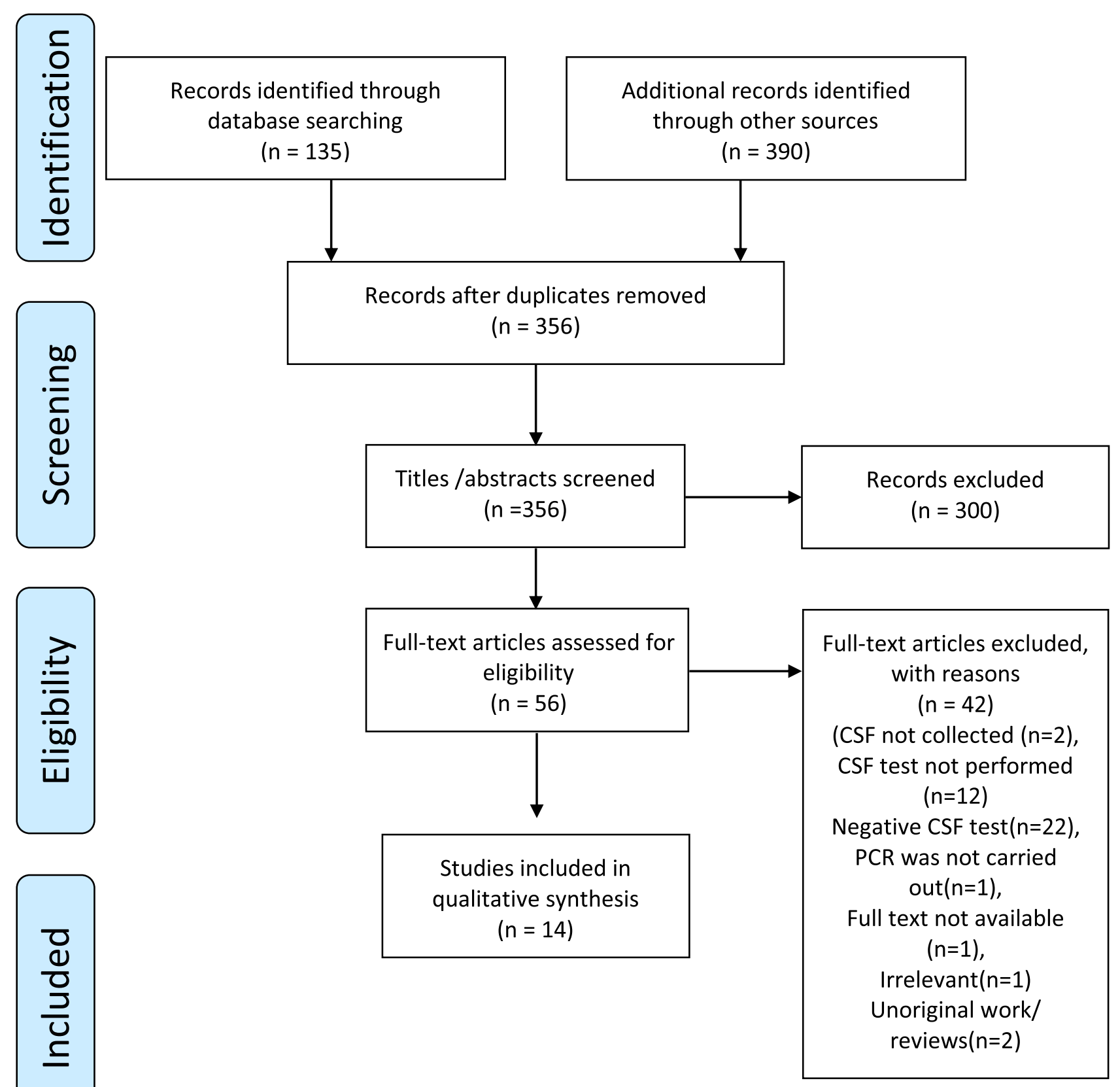

Figure I PRISMA flow diagram of literature search and selection.

Note: Adapted from Page MJ, McKenzie JE, Bossuyt PM, Boutron I, Hoffmann TC, Mulrow CD, et al. The PRISMA 2020 statement: an updated guideline for reporting systematic reviews. BMJ 2021;372:n7I. doi: 10.1136/bmj.n7I. ${ }^{39}$

\section{Radiological Findings}

Radiological findings (CXR, chest CT, systemic CT, Brain MRI, and head CT) were reported for $11 / 14$ patients. However, we could not extract the data from one cohort study. ${ }^{26}$ Radiological findings were normal in 2/14 patients. $^{18,28}$ The most common findings on brain MRI FLAIR were hyperintense regions in different areas of the brain (6/14), ${ }^{10,17,19-21,23}$ and the commonest finding on chest
CT was ground glass opacities in the lungs $(5 / 14)^{10,17,20,22,23}$ (Table 4).

\section{EEG Findings}

EEG findings were reported in two studies, ${ }^{20,28}$ two of which noted a similar generalised slowing of waves with no epileptic activity. ${ }^{20,28}$ One of these patients was noted to have a previous seizure. ${ }^{28}$ 
Table I Characteristics of Included Studies

\begin{tabular}{|c|c|c|c|c|c|c|c|}
\hline Study & Authors & Country & Research Design & $\begin{array}{l}\text { Sample } \\
\text { Size }\end{array}$ & $\begin{array}{c}\text { Number of CSF-Positive } \\
\text { Cases }\end{array}$ & Gender & Age \\
\hline I & Destras et $\mathrm{al}^{24}$ & France & $\begin{array}{c}\text { Retrospective Cohort } \\
\text { Study }\end{array}$ & 555 & 2 & $\mathrm{~N} / \mathrm{A}$ & Adults \\
\hline 2 & Huang et $\mathrm{al}^{28}$ & USA & Case Report & I & I & $\mathrm{F}$ & 40 \\
\hline 3 & Cebrián et al ${ }^{16}$ & Spain & Case Report & I & I & $\mathrm{F}$ & 74 \\
\hline 4 & Domingues et $\mathrm{al}^{18}$ & Brazil & Case Report & I & I & $\mathrm{F}$ & 42 \\
\hline 5 & Moriguchi et al ${ }^{17}$ & Japan & Case Report & I & I & M & 24 \\
\hline 6 & Fadakar et al ${ }^{19}$ & Iran & Case Report & I & 1 & M & 47 \\
\hline 7 & $\begin{array}{l}\text { Demirci Otluoglu } \\
\text { et }^{10}{ }^{10}\end{array}$ & Turkey & Case Report & I & 1 & M & 48 \\
\hline 8 & Helms et $\mathrm{al}^{25}$ & French & Cohort Study & 140 & I & $\mathrm{N} / \mathrm{A}$ & N/A \\
\hline 9 & Rostami et $\mathrm{al}^{20}$ & Sweden & Case Report & I & I & $\mathrm{F}$ & 55 \\
\hline 10 & Al-olama et $\mathrm{al}^{2 \mathrm{I}}$ & UAE & Case Report & I & I & M & 36 \\
\hline 11 & Mardani et $\mathrm{al}^{22}$ & Iran & Case Report & I & I & $\mathrm{F}$ & 64 \\
\hline 12 & Kremer et al ${ }^{26}$ & France & Retrospective Cohort & 28 & I & $\mathrm{N} / \mathrm{A}$ & N/A \\
\hline 13 & Westhoff et $\mathrm{al}^{23}$ & Germany & Case Report & I & I & $M$ & 69 \\
\hline
\end{tabular}

Abbreviations: CSF, cerebrospinal fluid; N/A, not available; M, male; F, female.

\section{Management and Treatment}

The management of 4 patients was not discussed in their respective studies, ${ }^{18,24-26}$ while the management for the remaining patients varied. Invasive intervention was required in two patients: Surgery was performed on 1/14 patients to remove the chronic subdural haematoma ${ }^{21}$ and endotracheal intubation and mechanical ventilation was required on another patient with impaired consciousness. ${ }^{17}$ The mainstay initial management in $4 / 14$ patients was acyclovir. ${ }^{10,20,22,28}$ This was, however, discontinued in one patient following negative herpes simplex virus results. ${ }^{28}$ Levetiracetam was given in $3 / 14$ patients ${ }^{10,23,28}$ and hydroxychloroquine was administered to $5 / 14$ patients. ${ }^{10,16,22,23,28}$ Table 5 shows the management and outcomes of the 14 SARS-CoV-2 CSF positive patients.

\section{Clinical Outcomes}

The outcomes at the end of the study periods varied in these 14 SARS-CoV-2 CSF positive patients (Table 5). Overall, 2/14 deaths ${ }^{24}$ and 4/14 ICU admissions ${ }^{17,21,25}$ were reported. Symptoms improved in $1 / 14$ cases who remained admitted, ${ }^{10}$ while $6 / 14$ cases were discharged/ recovered, ${ }^{16,18-20,23,28}$ and $1 / 14$ was transferred to a rehabilitation centre. ${ }^{20}$ Outcomes were not stated for two of the 14 patients. ${ }^{22,26}$

\section{Risk of Bias Across Studies}

Bias assessment is documented in Appendix 1, Tables S1 and S2.

\section{Discussion}

In this systematic review, we identified 14 articles which described 14 patients with positive SARS-CoV-2 CSF out of 733 articles. We systematically reviewed all reports of RTPCR positive SARS-CoV-2 CSF samples in the literature since the start of the outbreak in December. A mixedmethods exploratory approach was adopted for data analysis, making observations, and investigating any preliminary patterns and theories that can be extracted from the sporadic cases reported. Common symptoms were headache fever, vomiting, cough, visual disturbances, diarrhoea, and seizures. Four patients were admitted to ICU, one was admitted to a rehabilitation facility, and two died.

The paucity of case reports that reported CSF-positive SARS-CoV-2 patients may indicate that viral neuroinvasion by SARS-CoV-2 appears to be rare. This is in 
Table 2 Summary of Presentation and Clinical Course of All Cases Testing Positive for SARS-CoV-2 in CSF Samples

\begin{tabular}{|c|c|c|c|c|c|c|}
\hline Study & Authors & $\begin{array}{c}\text { NP } \\
\text { Result }\end{array}$ & $\begin{array}{l}\text { Positive } \\
\text { CSF } \\
\text { Result }\end{array}$ & $\begin{array}{l}\text { General } \\
\text { Signs }\end{array}$ & Neural Signs and Symptoms & $\begin{array}{l}\text { Respiratory } \\
\text { Symptoms }\end{array}$ \\
\hline 1 & $\begin{array}{l}\text { Destras } \\
\text { et } \mathrm{al}^{24}\end{array}$ & Positive & $2 / 555$ & $\mathrm{~N} / \mathrm{A}$ & N/A & N/A \\
\hline 2 & $\begin{array}{l}\text { Huang } \\
\text { et } \mathrm{al}^{28}\end{array}$ & Positive & I & $\begin{array}{l}\text { Fever; } \\
\text { Lethargy }\end{array}$ & $\begin{array}{l}\text { Headache; Seizures; Photophobia; Impaired consciousness; } \\
\qquad \text { Neck stiffness }\end{array}$ & None \\
\hline 3 & $\begin{array}{l}\text { Cebrián } \\
\text { et al }\end{array}$ & Positive & I & $\begin{array}{l}\text { Myalgia; } \\
\text { Nausea; } \\
\text { Vomiting }\end{array}$ & $\begin{array}{l}\text { Headache; Photophobia; Visual disturbance (blurred } \\
\text { binocular vision); Incoherent speech }\end{array}$ & None \\
\hline 4 & $\begin{array}{l}\text { Domingues } \\
\text { et }^{18}{ }^{18}\end{array}$ & Negative & I & Diarrhoea & $\begin{array}{l}\text { Paresthesia and hypoesthesia of the left upper limb; Left } \\
\text { hemithorax and hemiface }\end{array}$ & $\begin{array}{l}\text { Common cold; } \\
\qquad \text { Nasal } \\
\text { obstruction }\end{array}$ \\
\hline 5 & $\begin{array}{l}\text { Moriguchi } \\
\text { et al }\end{array}$ & Negative & I & Fever; Fatigue & $\begin{array}{l}\text { Headache; Seizures (transient generalised); Impaired } \\
\text { consciousness }\end{array}$ & Sore throat \\
\hline 6 & $\begin{array}{l}\text { Fadakar } \\
\text { et al }\end{array}$ & Positive & I & $\begin{array}{l}\text { Myalgia; } \\
\text { Fatigue }\end{array}$ & $\begin{array}{l}\text { Headache; Visual disturbances (saccade eye movements, } \\
\text { optokinetic and end gaze rotational nystagmus); Gait } \\
\text { disturbances; Vertigo }\end{array}$ & Cough \\
\hline 7 & $\begin{array}{l}\text { Demirci } \\
\text { Otluoglu } \\
\text { et } \text { al }^{10}\end{array}$ & $\mathrm{~N} / \mathrm{A}$ & I & $\begin{array}{l}\text { Myalgia; } \\
\text { Fatigue }\end{array}$ & Headache; Neck stiffness; Anosmia & Cough \\
\hline 8 & $\begin{array}{l}\text { Helms } \\
\text { et } \mathrm{al}^{25}\end{array}$ & Positive & $1 / 140$ & $\mathrm{~N} / \mathrm{A}$ & $\mathrm{N} / \mathrm{A}$ & $\begin{array}{l}\text { Acute } \\
\text { respiratory } \\
\text { distress } \\
\text { syndrome }\end{array}$ \\
\hline 9 & $\begin{array}{l}\text { Rostami } \\
\text { et } \mathrm{al}^{20}\end{array}$ & Positive & I & Fever; Myalgia & Impaired brain stem reflexes & None \\
\hline 10 & $\begin{array}{c}\text { Al-olama } \\
\text { et } \mathrm{al}^{21}\end{array}$ & Positive & I & $\begin{array}{l}\text { Fever; Myalgia, } \\
\text { Diarrhoea; } \\
\text { Vomiting }\end{array}$ & Headache; Impaired consciousness & $\begin{array}{c}\text { Cough; } \\
\text { Pharyngitis }\end{array}$ \\
\hline II & $\begin{array}{c}\text { Mardani } \\
\text { et } \mathrm{al}^{22}\end{array}$ & Positive & I & $\begin{array}{c}\text { Generalised } \\
\text { weakness }\end{array}$ & Impaired consciousness & $\begin{array}{l}\text { Acute } \\
\text { progressive } \\
\text { dyspnoea }\end{array}$ \\
\hline 12 & $\begin{array}{l}\text { Kremer } \\
\text { et } \mathrm{al}^{26}\end{array}$ & $N / A$ & $1 / 28$ & $\mathrm{~N} / \mathrm{A}$ & $\mathrm{N} / \mathrm{A}$ & $\mathrm{N} / \mathrm{A}$ \\
\hline 13 & $\begin{array}{c}\text { Westhoff } \\
\text { et } \mathrm{al}^{23}\end{array}$ & Positive & I & $\begin{array}{c}\text { Fever; } \\
\text { Diarrhoea }\end{array}$ & Seizures (convulsive); Left-sided neglect & Cough \\
\hline
\end{tabular}

Abbreviations: N/A, not available; CSF, cerebrospinal fluid.

accordance with another systematic review in which $6 \%{ }^{17}$ tested positive out of 304 patients whose CSF was tested for SARS-CoV-2. ${ }^{29}$

The low prevalence of CSF positive SARS-CoV-2 results can be attributed to several factors, the first is that CSF testing rate was initially low since it is done only in cases with serious CNS manifestations, if patients had no CNS manifestations they would therefore not be tested. Secondly, isolation of SARS-CoV-2 in CSF may be challenging because of rapid CSF clearance, low titters or delayed sampling. ${ }^{30-32}$ Further, CSF antibodies test was not always done for CSF negative patients which could 
Table 3 Blood and Cerebrospinal Fluid Lab Findings of Cases with SARS-CoV-2 Positive CSF Samples

\begin{tabular}{|c|c|c|c|c|c|c|c|c|}
\hline \multirow[t]{3}{*}{ Study } & \multirow[t]{3}{*}{ Author } & \multicolumn{7}{|c|}{ Laboratory Findings } \\
\hline & & \multicolumn{4}{|c|}{ CSF Sample } & \multicolumn{3}{|c|}{ Blood Sample } \\
\hline & & RBC & WBC & Protein & Glu & WCC & Glu & CRP \\
\hline I & Destras et $\mathrm{al}^{24}$ & \multicolumn{7}{|c|}{ Data could not be extracted } \\
\hline 2 & Huang et $\mathrm{al}^{28}$ & $\uparrow$ & N/A & $\uparrow$ & $*$ & $\uparrow(100 \%$ lymphocytes $)$ & $*$ & $\mathrm{~N} / \mathrm{A}$ \\
\hline 3 & Cebrián et al $^{16}$ & N/A & $N / A$ & N/A & $N / A$ & $\uparrow$ & $N / A$ & $\leftrightarrow$ \\
\hline 4 & Domingues et al $^{18}$ & N/A & $\leftrightarrow$ & $\leftrightarrow$ & $\leftrightarrow$ & N/A & N/A & $\leftrightarrow$ \\
\hline 5 & Moriguchi et al ${ }^{17}$ & $\stackrel{\leftrightarrow}{\text { None }}$ & $\begin{array}{l}\uparrow(10 \mathrm{MN} * * \\
\left.2 \mathrm{PMN}^{* * *}\right)\end{array}$ & $\mathrm{N} / \mathrm{A}$ & N/A & $\uparrow$ (Neutrophil Predominant) & $\mathrm{N} / \mathrm{A}$ & $\uparrow$ \\
\hline 6 & Fadakar et al ${ }^{19}$ & $\mathrm{~N} / \mathrm{A}$ & N/A & $N / A$ & $N / A$ & $\leftrightarrow(32 \%$ lymphocytes $)$ & N/A & $\leftrightarrow$ \\
\hline 7 & 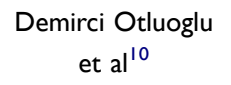 & N/A & $\leftrightarrow$ & $\leftrightarrow$ & $\uparrow$ & $\begin{array}{c}\leftrightarrow(24.4 \% \text { lymphocytes, } 62.8 \% \\
\text { Neutrophils })\end{array}$ & $\underset{L^{* * * *}}{105 \mathrm{mmol} /}$ & $\leftrightarrow$ \\
\hline 8 & Helms et $\mathrm{al}^{25}$ & \multicolumn{7}{|c|}{ Data could not be extracted. } \\
\hline 9 & Rostami et $\mathrm{al}^{20}$ & N/A & $\mathrm{N} / \mathrm{A}$ & $\mathrm{N} / \mathrm{A}$ & N/A & $\leftrightarrow / \uparrow$ & N/A & $\leftrightarrow / \uparrow$ \\
\hline 10 & Al-olama et $\mathrm{al}^{2 \mathrm{I}}$ & N/A & $\mathrm{N} / \mathrm{A}$ & N/A & N/A & $\uparrow$ & $\uparrow$ & $\leftrightarrow$ \\
\hline 11 & Mardani et $\mathrm{al}^{22}$ & $N / A$ & $\begin{array}{c}\uparrow(90 \% \\
\text { polymorph) }\end{array}$ & $\uparrow$ & $\downarrow$ & $\uparrow$ Polymorphs > lymphocytes & $\uparrow$ & $\mathrm{N} / \mathrm{A}$ \\
\hline 12 & Kremer et $\mathrm{al}^{26}$ & \multicolumn{7}{|c|}{ Data could not be extracted. } \\
\hline 13 & Westhoff et al ${ }^{23}$ & $\mathrm{~N} / \mathrm{A}$ & $\begin{array}{c}\leftrightarrow(100 \% \\
\text { lymphocytes })\end{array}$ & $\uparrow$ & $\leftrightarrow$ & $\downarrow$ Lymphopenia & $N / A$ & $\uparrow$ \\
\hline
\end{tabular}

Notes: *Units Not Reported, **Polymorphonuclear, ***Mononuclear. ****Non-fasting blood glucose.

Abbreviations: RBC, red blood cells; WBC, white blood cells; Glu, glucose; WCC, white cell count; CRP, C-reactive protein; N/A, not available; $\uparrow$, elevated levels; $\downarrow$, decreased levels; $\leftrightarrow$, normal levels.

have led to missing resolved infection. In a recent systematic review, it was reported that out of those who did not test positive for CSF but had CNS symptoms, 42/58 $(72 \%)$ tested positive for SARS-CoV-2 antibodies in the $\mathrm{CSF}^{29}$

The discrepancy between CSF results and NP results could be attributed to the variability in the cycle threshold $\mathrm{Ct}(\mathrm{Ct}$; the number of amplification cycles required for the target gene to exceed the threshold) cut-off point (some used 40, some 37 and some 35). ${ }^{17,29}$ Preanalytical issues such as collection techniques, and inadequate sample storage/transportation, timing of sample throughout the course of the disease which could have led to serious diagnostic errors. ${ }^{33}$ Further, serum antibodies were not always checked for NP PCR negative patients which could have verified the resolved infections. Additionally, CSF SARS-CoV-2 PCR testing is not $100 \%$ specific for intrathecal virus, in part because a sample can be contaminated from shed airborne virus or blood contamination. ${ }^{34}$ Interestingly, PCR testing for the N2 gene target of SARS-CoV-2 was noted to have the highest sensitivity in CSF when compared with a nasopharyngeal swab, bronchoalveolar lavage, sputum, plasma, or stool. ${ }^{37}$ Despite this, the clinical indications for performing LPs in patients with SARS-CoV-2 infection remain unclear. Additionally, how clinicians can use information gained from LPs, such as cell counts and infectious workup, in the management of COVID-19 and neurological symptoms has not been established.

In this review, comorbidities were not commonly present among CSF positive patients (60\%) which reemphasizes that otherwise healthy individuals may present with CSF viral neuro-infiltration in the absence of comorbidities in the setting of COVID-19. ${ }^{35}$

Patients whose CSF samples tested positive for SARS$\mathrm{CoV}-2$ reported a range of symptoms, with respiratory distress not always being reported. Headache, fever, 
Table 4 Radiological Findings of Cases with SARS-CoV-2 Positive CSF Samples

\begin{tabular}{|c|c|c|c|c|}
\hline Study & Authors & Chest Findings & Brain MRI & Head CT \\
\hline 1 & $\begin{array}{l}\text { Destras } \\
\text { et } \mathrm{al}^{24}\end{array}$ & $\mathrm{~N} / \mathrm{A}$ & N/A & $\mathrm{N} / \mathrm{A}$ \\
\hline 2 & $\begin{array}{l}\text { Huang } \\
\text { et } \mathrm{al}^{28}\end{array}$ & Unremarkable. & $\mathrm{N} / \mathrm{A}$ & Unremarkable. \\
\hline 3 & $\begin{array}{l}\text { Cebrián } \\
\text { et al }\end{array}$ & Unremarkable. & $\begin{array}{l}\text { Right parietal cortical-subcortical restricted } \\
\text { diffusion }\end{array}$ & Unremarkable. \\
\hline 4 & $\begin{array}{l}\text { Domingues } \\
\text { et al }\end{array}$ & Unremarkable. & Unremarkable. & Unremarkable. \\
\hline 5 & $\begin{array}{l}\text { Moriguchi } \\
\text { et al }{ }^{17}\end{array}$ & Ground glass opacities & $\begin{array}{l}\text { Hyperintense lesions in the right mesial } \\
\text { temporal lobe and hippocampus; Slight } \\
\text { hippocampal atrophy. }\end{array}$ & Unremarkable. \\
\hline 6 & $\begin{array}{l}\text { Fadakar } \\
\text { et al }{ }^{19}\end{array}$ & $N / A$ & $\begin{array}{l}\text { Bilateral cerebellar hemispheres and vermis } \\
\text { hyperintensities; Edema; Cortical-meningeal } \\
\text { enhancement of cerebellum }\end{array}$ & $\mathrm{N} / \mathrm{A}$ \\
\hline 7 & $\begin{array}{l}\text { Demirci } \\
\text { Otluoglu } \\
\text { et al }\end{array}$ & $\begin{array}{l}\text { Ground glass opacities; } \\
\text { Consolidation }\end{array}$ & $\begin{array}{l}\text { Hyperintense lesions in the posterior } \\
\text { medial cortical surface of the temporal } \\
\text { lobe; Hyperintense lesions in the upper } \\
\text { cervical spinal cord. }\end{array}$ & Unremarkable. \\
\hline 8 & $\begin{array}{l}\text { Helms } \\
\text { et } \mathrm{al}^{25}\end{array}$ & $N / A$ & N/A & $\mathrm{N} / \mathrm{A}$ \\
\hline 9 & $\begin{array}{l}\text { Rostami } \\
\text { et } \mathrm{al}^{20}\end{array}$ & $\begin{array}{l}\text { Ground Glass opacities/ } \\
\text { consolidations. }\end{array}$ & $\begin{array}{l}\text { Ist Brain MRI: Acute necrotizing } \\
\text { encephalitis. } \\
\text { 2nd Brain MRI: Partial regression of the } \\
\text { changes in the brainstem and medial } \\
\text { temporal lobes; More pronounced } \\
\text { hyperintensities in central thalami and } \\
\text { subinsular regions. }\end{array}$ & $\begin{array}{l}\text { Symmetrical hypodensities in the thalami; } \\
\text { Low attenuation areas in the thalami and } \\
\text { midbrain. }\end{array}$ \\
\hline 10 & $\begin{array}{l}\text { Al-olama } \\
\text { et } \mathrm{al}^{21}\end{array}$ & Unremarkable & $\begin{array}{l}\text { Right frontal intracerebral hematoma; } \\
\text { Subarachnoid hemorrhage in the ipsilateral } \\
\text { sylvian fissure and frontal and temporal } \\
\text { lobes; Acute subdural hematoma; Edema } \\
\text { causing midline shift. }\end{array}$ & $\begin{array}{l}\text { Hyperintensities in the bilateral } \\
\text { supratentorial leptomeningeal area; } \\
\text { Chronic right subdural hematoma; Re- } \\
\text { reabsorbing intracerebral hematoma; } \\
\text { Perilesional brain edema causing midline } \\
\text { shift. }\end{array}$ \\
\hline II & $\begin{array}{l}\text { Mardani } \\
\text { et } \mathrm{al}^{22}\end{array}$ & $\begin{array}{l}\text { Bilateral Pleural effusion; } \\
\text { Collapse consolidation of } \\
\text { basal segments; Patchy } \\
\text { ground-glass opacities }\end{array}$ & N/A & N/A \\
\hline 12 & $\begin{array}{l}\text { Kremer } \\
\text { et } \mathrm{al}^{26}\end{array}$ & & Data could not be extracted & \\
\hline 13 & $\begin{array}{l}\text { Westhoff } \\
\text { et } \mathrm{al}^{23}\end{array}$ & $\begin{array}{l}\text { Ground-glass opacities; } \\
\text { Consolidation }\end{array}$ & $\begin{array}{l}\text { Linear meningeal hyperintensities; White } \\
\text { matter edema }\end{array}$ & N/A \\
\hline
\end{tabular}

vomiting, coughing, and visual disturbances were commonly reported, before progressing to more severe/intense neurological symptoms. ${ }^{36}$ This might have an implication on CSF testing for diagnostic purposes. Further studies are required to define whether CSF SARS-CoV-2 testing is warranted in certain clinical contexts. ${ }^{16,20,28}$ 
Table 5 The Management and Outcomes of SARS-CoV-2 CSF Positive Patients

\begin{tabular}{|c|c|c|c|c|c|c|}
\hline Study & Authors & Antivirals & Antibiotics & Antiepileptics & Other Medications & Outcomes \\
\hline I & $\begin{array}{l}\text { Destras } \\
\text { et } \mathrm{al}^{24}\end{array}$ & N/A & N/A & N/A & N/A & Death (2) \\
\hline 2 & $\begin{array}{l}\text { Huang } \\
\text { et } \mathrm{al}^{28}\end{array}$ & Acyclovir & Ceftriaxone/Vancomycin & Levetiracetam & $\mathrm{HCQ}$ & Full Recovery \\
\hline 3 & $\begin{array}{l}\text { Cebrián } \\
\text { et } \mathrm{al}^{16}\end{array}$ & $\begin{array}{l}\text { Lopinavir/ } \\
\text { Ritonavir }\end{array}$ & Ceftriaxone & None & $\begin{array}{l}\text { Pain drugs; Fluid replacement; Oxygen } \\
\text { therapy; HCQ; Acetaminophen; } \\
\text { Dexketoprofen; Acetylsalicylic acid }\end{array}$ & Discharged \\
\hline 4 & 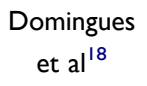 & N/A & $\mathrm{N} / \mathrm{A}$ & $\mathrm{N} / \mathrm{A}$ & N/A & Full recovery \\
\hline 5 & $\begin{array}{l}\text { Moriguchi } \\
\text { et al }{ }^{17}\end{array}$ & $\begin{array}{l}\text { Aciclovir; } \\
\text { Favipiravir }\end{array}$ & $\begin{array}{l}\text { Ceftriaxone; } \\
\text { Vancomycin }\end{array}$ & Levetiraceta & $\begin{array}{l}\text { Endotracheal intubation + Mechanical } \\
\text { ventilation; Steroids }\end{array}$ & ICU \\
\hline 6 & $\begin{array}{l}\text { Fadakar } \\
\text { et al }\end{array}$ & $\begin{array}{l}\text { Lopinavir; } \\
\text { Ritonavir }\end{array}$ & None & None & None & Discharged \\
\hline 7 & $\begin{array}{l}\text { Demirci } \\
\text { Otluoglu } \\
\text { et } \text { al }^{10}\end{array}$ & $\begin{array}{l}\text { Favipiravir; } \\
\text { Acyclovir }\end{array}$ & Piperacillin/Tazobactam & Levetiracetam & HCQ; Steroids & $\begin{array}{l}\text { Stable under } \\
\text { treatment }\end{array}$ \\
\hline 8 & $\begin{array}{l}\text { Helms } \\
\text { et } \mathrm{al}^{25}\end{array}$ & $\mathrm{~N} / \mathrm{A}$ & $N / A$ & N/A & N/A & ICU \\
\hline 9 & $\begin{array}{l}\text { Rostami } \\
\text { et } \mathrm{al}^{20}\end{array}$ & Acyclovir & None & None & $\begin{array}{l}\text { IVIG; Immunotherapy with plasma } \\
\text { exchange }\end{array}$ & $\begin{array}{l}\text { Discharged; } \\
\text { Rehabilitation }\end{array}$ \\
\hline 10 & $\begin{array}{l}\text { Al-olama } \\
\text { et } \mathrm{al}^{21}\end{array}$ & & None & None & Burr hole & ICU \\
\hline II & $\begin{array}{l}\text { Mardani } \\
\text { et } \mathrm{al}^{22}\end{array}$ & $\begin{array}{l}\text { Lopinavir/ } \\
\text { Ritonavir; } \\
\text { Acyclovir }\end{array}$ & $\begin{array}{c}\text { Ceftriaxone; } \\
\text { Clindamycin; } \\
\text { Meropenem; } \\
\text { Vancomycin; Ampicillin }\end{array}$ & None & $\begin{array}{l}\text { HCQ; Steroids; Folinic acid; Fluorouracil; } \\
\text { Irinotecan }\end{array}$ & N/A \\
\hline 12 & $\begin{array}{l}\text { Kremer } \\
\text { et }\left.^{26}\right|^{26}\end{array}$ & N/A & N/A & N/A & N/A & N/A \\
\hline 13 & $\begin{array}{l}\text { Westhoff } \\
\text { et } \mathrm{al}^{23}\end{array}$ & None & None & Levetiracetam & $\begin{array}{l}\text { Steroids; Insulin; Oxygen supply; } \\
\text { Tacrolimus; HCQ }\end{array}$ & $\begin{array}{l}\mathrm{ICU} \rightarrow \text { full } \\
\text { recovery }\end{array}$ \\
\hline
\end{tabular}

Abbreviations: N/A, not available; HCQ, hydroxychloroquine; IVIG, intravenous immunoglobulin G; ICU, intensive care unit.

High levels of lymphocytes and protein were reported in 2/14 and 3/14 CSF samples of COVID-19 positive patients, respectively. CSF Pleocytosis is expected and occurs secondary to an inflammatory and/or infectious process. ${ }^{29}$ Also, the observation of hyperproteinorrachia may indicate axonal injury and the existence of intrathecal antibodies. ${ }^{29}$ There were no striking blood findings except for leucocytosis and hyperglycaemia. Both are expected and reflects the inflammatory process due to the disease. However, due to the lack of data, it is not known whether patients with hyperglycaemia in these case reports had pre-existing diabetes or not.
The EEG findings showed two patients with generalised slowing of waves with no epileptic activity. One of them had a history of pre-existing seizures. It is well documented in the literature that COVID-19 can result in EEG changes, and it is correlated with disease severity. ${ }^{37,38}$ EEG findings in COVID-19 may indicate localized dysfunction, non-specific encephalopathy, and cortical irritability. ${ }^{38}$ In fact, frontal findings are common and have been proposed as a biomarker for COVID-19 encephalopathy. ${ }^{38}$ Diffuse EEG changes in the context of COVID-19 have been speculated to result from systemic involvement or diffuse viral 
involvement of the brain while frontal EEG findings suggest direct brain involvement. ${ }^{38}$

There are several limitations to this review mainly attributed to lack of data from original case series such as description of test technique, time at which sample is collected, CSF analysis and SARS CoV antibodies. Lastly, this review is limited by publication bias and the paucity of published case reports.

More studies are needed to describe how results of LP influence clinical decision-making in a case series of patients with COVID-19 even if SARS-CoV-2 is not detected in the CSF.

\section{Conclusions}

This review describes the unique characteristics of patients who tested positive for SARS-CoV-2 in their CSF sample, regardless of the test outcome of the NP sample. Nevertheless, there are not enough data in the literature for guideline formation, especially given the fact that COVID-19 is a novel virus and an emergent crisis. Hence, more evidence is needed to improve our understanding regarding how results of LP influence clinical decision-making in a case series of patients with COVID-19 even if SARS-CoV-2 is not detected in the CSF. Additionally, how clinicians can use information gained from LPs, such as cell counts and infectious workup, in the management of COVID-19.

\section{Abbreviations}

COVID-19, coronavirus disease 2019; CSF, cerebrospinal fluid; NP, nasopharyngeal; RT-PCR, reverse transcriptase polymerase chain reaction; LP, Lumbar Puncture; PNS, peripheral nervous system; NIH, National Institutes of Health; JBI, Joanna Briggs Institute; PRISMA, Preferred Reporting Items for Systematic Reviews and MetaAnalyses.

\section{Data Sharing Statement}

All data generated or analysed during this study are included in this published article and its supplementary information files.

\section{Acknowledgments}

We would like to thank Mr. Niall O'Brien for assisting us with our search strategy.

\section{Author Contributions}

MS conceived of the idea. MS, SIM, WK, LRM, and YA drafted one or more sections of the manuscript. MS, SIM, and GAJ reviewed and edited the manuscript. GAJ supervised the work. All authors made a significant contribution to the work reported, whether that is in the conception, study design, execution, acquisition of data, analysis and interpretation, or in all these areas; took part in drafting, revising or critically reviewing the article; gave final approval of the version to be published; have agreed on the journal to which the article has been submitted; and agree to be accountable for all aspects of the work.

\section{Funding}

Publication cost was funded by the Royal College of Surgeons in Ireland. Medical University of Bahrain.

\section{Disclosure}

The authors declare no conflicts of interest in this work.

\section{References}

1. World Health Organization Organisation TWH. Q\&A on coronaviruses (COVID-19); April 17, 2020. Available from: https://www. who.int/emergencies/diseases/novel-coronavirus-2019/question-andanswers-hub/q-a-detail/q-a-coronaviruses. Accessed December 7, 2021.

2. Ellul MA, Benjamin L, Singh B, et al. Neurological associations of COVID-19. Lancet Neurol. 2020;19(9):767-83\{Ellul, 2020 \#17\}. doi:10.1016/S1474-4422(20)30221-0

3. Chen X, Laurent S, Onur OA, et al. A systematic review of neurological symptoms and complications of COVID-19. J Neurol. 2021;268(2):392-402.

4. Baig AM, Khaleeq A, Ali U, Syeda H. Evidence of the COVID-19 virus targeting the CNS: tissue distribution, host-virus interaction, and proposed neurotropic mechanisms. ACS Chem Neurosci. 2020;11 (7):995-998. doi:10.1021/acschemneuro.0c00122

5. Cain MD, Salimi H, Diamond MS, Klein RS. Mechanisms of pathogen invasion into the central nervous system. Neuron. 2019;103 (5):771-783. doi:10.1016/j.neuron.2019.07.015

6. Baig AM. Emerging insights for better delivery of chemicals and stem cells to the brain. ACS Chem Neurosci. 2017;8(6):1119-1121. doi:10.1021/acschemneuro.7b00106

7. Netland J, Meyerholz DK, Moore S, Cassell M, Perlman S. Severe acute respiratory syndrome coronavirus infection causes neuronal death in the absence of encephalitis in mice transgenic for human ACE2. J Virol. 2008;82(15):7264-7275. doi:10.1128/JVI.00737-08

8. Desforges M, Le Coupanec A, Dubeau P, et al. Human coronaviruses and other respiratory viruses: underestimated opportunistic pathogens of the central nervous system? Viruses. 2019;12(1):14. doi:10.3390/ v12010014

9. Bohmwald K, Gálvez NMS, Ríos M, Kalergis AM. Neurologic alterations due to respiratory virus infections. Front Cell Neurosci. 2018;12:386. doi:10.3389/fncel.2018.00386

10. Demirci Otluoglu G, Yener U, Demir MK, Yilmaz B. Encephalomyelitis associated with covid-19 infection: case report. Br J Neurosurg. 2020;1-3. doi:10.1080/02688697.2020.1787342 
11. Burki TK. Testing for COVID-19. Lancet Respir Med. 2020;8(7): e63-e64. doi:10.1016/S2213-2600(20)30247-2

12. Prevention CfDCa. CDC 2019-Novel Coronavirus (2019-nCoV) real-time RT-PCR diagnostic panel; July 13, 2020 [updated July 13, 2020]. Available from: https://www.fda.gov/media/134922/download. Accessed December 7, 2021.

13. World Health Organization (WHO) TWHO. Protocol: real-time RT-PCR assays for the detection of SARS-CoV-2; 2020. Available from: https://www.who.int/docs/default-source/coronaviruse/realtime-rt-pcr-assays-for-the-detection-of-sars-cov-2-institut-pasteurparis.pdf?sfvrsn=3662fcb6_2. Accessed December 7, 2021.

14. Doherty CM, Forbes RB. Diagnostic lumbar puncture. Ulster Med J. 2014;83(2):93-102.

15. Wu Y, Xu X, Chen $\mathrm{Z}$, et al. Nervous system involvement after infection with COVID-19 and other coronaviruses. Brain Behav Immun. 2020;87:18-22. doi:10.1016/j.bbi.2020.03.031

16. Cebrián J, Gonzalez-Martinez A, García-Blanco MJ, et al. Headache and impaired consciousness level associated with SARS-CoV-2 in CSF: a case report. Neurology. 2020;95(6):266-268. doi:10.1212/ WNL.0000000000010213

17. Moriguchi T, Harii N, Goto J, et al. A first case of meningitis/ encephalitis associated with SARS-coronavirus-2. Int J Infect Dis. 2020;94:55-58. doi:10.1016/j.ijid.2020.03.062

18. Domingues RB, Mendes-Correa MC, de Moura Leite FBV, et al. First case of SARS-COV-2 sequencing in cerebrospinal fluid of a patient with suspected demyelinating disease. $J$ Neurol. 2020;267 (11):3154-3156. doi:10.1007/s00415-020-09996-w

19. Fadakar N, Ghaemmaghami S, Masoompour SM, et al. A first case of acute cerebellitis associated with Coronavirus Disease (COVID-19): a case report and literature review. Cerebellum. 2020:1-4. doi:10.1007/s12311-019-01083-9

20. Rostami E, Virhammar J, Kumlien E, et al. Data from: acute necrotizing encephalopathy with SARS-CoV-2 RNA confirmed in cerebrospinal fluid. Dryad. 2020;95(10):445-449.

21. Al-olama M, Rashid A, Garozzo D. COVID-19-associated meningoencephalitis complicated with intracranial hemorrhage: a case report. Acta Neurochir. 2020;162(7):1495-1499. doi:10.1007/ s00701-020-04402-w

22. Mardani M, Nadji SA, Sarhangipor KA, Sharifi-Razavi A, Baziboroun M. COVID-19 infection recurrence presenting with meningoencephalitis. New Microbes New Infect. 2020;37:100732. doi:10.1016/j.nmni.2020.100732

23. Westhoff TH, Seibert FS, Bauer F, et al. Allograft infiltration and meningoencephalitis by SARS-CoV-2 in a pancreas-kidney transplant recipient. Am J Transplant. 2020;20(11):3216-3220. doi:10.1111/ajt.16223

24. Destras G, Bal A, Escuret V, Morfin F, Lina B, Josset L. Systematic SARS-CoV-2 screening in cerebrospinal fluid during the COVID-19 pandemic. Lancet Microbe. 2020;1(4):e149. doi:10.1016/S26665247(20)30066-5

25. Helms J, Kremer S, Merdji H, et al. Delirium and encephalopathy in severe COVID-19: a cohort analysis of ICU patients. Crit Care. 2020;24(1):491. doi:10.1186/s13054-020-03200-1
26. Kremer S, Lersy F, de Sèze J, et al. Brain MRI findings in severe COVID-19: a retrospective observational study. Radiology. 2020;297 (2):E242-E251.

27. Duong L, Xu P, Liu A. Meningoencephalitis without respiratory failure in a young female patient with COVID-19 infection in downtown los angeles, early April 2020. Brain, Behavior, and Immunity. 2020;87:33. doi:10.1016/j.bbi.2020.04.024

28. Huang YH, Jiang D, Huang JT. SARS-CoV-2 detected in cerebrospinal fluid by PCR in a case of COVID-19 encephalitis. Brain Behav Immun. 2020;87:149. doi:10.1016/j.bbi.2020.05.012

29. Lewis A, Frontera J, Placantonakis DG, et al. Cerebrospinal fluid in COVID-19: a systematic review of the literature. J Neurol Sci. 2021;421:117316. doi:10.1016/j.jns.2021.117316

30. Zanin L, Saraceno G, Panciani PP, et al. SARS-CoV-2 can induce brain and spine demyelinating lesions. Acta Neurochir. 2020;162 (7):1491-1494. doi:10.1007/s00701-020-04374-x

31. Ye M, Ren Y, Lv T. Encephalitis as a clinical manifestation of COVID-19. Brain Behav Immun. 2020;88:945-946. doi:10.1016/j. bbi.2020.04.017

32. Panciani PP, Saraceno G, Zanin L, et al. SARS-CoV-2: "three-steps" infection model and CSF diagnostic implication. Brain Behav Immun. 2020;87:128-129. doi:10.1016/j.bbi.2020.05.002

33. Peñarrubia L, Ruiz M, Porco R, et al. Multiple assays in a real-time RT-PCR SARS-CoV-2 panel can mitigate the risk of loss of sensitivity by new genomic variants during the COVID-19 outbreak. Int J Infect Dis. 2020;97:225-229. doi:10.1016/j.ijid.2020.06.027

34. Needham EJ, Chou SH, Coles AJ, Menon DK. Neurological implications of COVID-19 infections. Neurocrit Care. 2020;32(3):667-671. doi:10.1007/s12028-020-00978-4

35. Lindan CE, Mankad K, Ram D, et al. Neuroimaging manifestations in children with SARS-CoV-2 infection: a multinational, multicentre collaborative study. Lancet Child Adolesc Health. 2021;5 (3):167-177. doi:10.1016/S2352-4642(20)30362-X

36. Alimohamadi Y, Sepandi M, Taghdir M, Hosamirudsari H. Determine the most common clinical symptoms in COVID-19 patients: a systematic review and meta-analysis. J Prev Med Hyg. 2020;61(3): E304-E312. doi:10.15167/2421-4248/jpmh2020.61.3.1530

37. Menon U, Fine L, Chimakurthy A, Khan F, Ramsay E. EEG findings in COVID-19 positive patients: a case series (4816). Neurology. 2021;96(15 Supplement):4816.

38. Antony AR, Haneef Z. Systematic review of EEG findings in 617 patients diagnosed with COVID-19. Seizure. 2020;83:234-241. doi:10.1016/j.seizure.2020.10.014

39. Page MJ, McKenzie JE, Bossuyt PM, Boutron I, Hoffmann TC, Mulrow CD, et al. The PRISMA 2020 statement: an updated guideline for reporting systematic reviews. BMJ. 2021;372:n71. doi:10.1136/bmj.n71
International Journal of General Medicine

\section{Publish your work in this journal}

The International Journal of General Medicine is an international, peer-reviewed open-access journal that focuses on general and internal medicine, pathogenesis, epidemiology, diagnosis, monitoring and treatment protocols. The journal is characterized by the rapid reporting of reviews, original research and clinical studies
Dovepress

across all disease areas. The manuscript management system is completely online and includes a very quick and fair peer-review system, which is all easy to use. Visit http://www.dovepress.com/ testimonials.php to read real quotes from published authors. 